\title{
Circumcision reduced the risk of contracting HIV infection in young sexually active Kenyan men
}

Bailey RC, Moses S, Parker CB, et al. Male circumcision for HIV prevention in young men in Kisumu, Kenya: a randomised controlled trial. Lancet 2007;369:643-56.

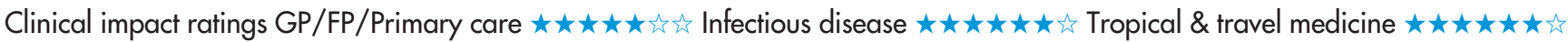
In young sexually active men in Kenya, does circumcision prevent the transmission of HIV infection?

\section{METHODS}

L

Design: randomised controlled trial.

Allocation: $\left\{\right.$ concealed $\left.^{*}\right\} \dagger$

Blinding: $\{$ blinded (outcome assessors) $\}+{ }^{*}$

Follow up period: median 24 months.

Setting: Kisumu district, Kenya

을 Participants: 2784 men who were 18-24 years of age (median age $20 \mathrm{y}$ ), were HIV negative and sexually active (median 4 partners over lifetime), had haemoglobin concentrations $\geqslant 90 \mathrm{~g} /$ I, and planned to stay in the Kisumu district for $\geqslant 2$ years. Exclusion criteria: foreskin covered less than half the glans, haemophilia or other bleeding disorder, high prothrombin time index, contraindications to surgery, or absolute indication for circumcision.

Intervention: circumcision ( $\mathrm{n}=1391$ ) or delayed circumcision (control; $n=1393$ ). Surgery was done under local anaesthesia in the study clinic, and men were given verbal and written instructions on postoperative wound care. Participants were advised to avoid sexual activity for $\geqslant 30$ days.

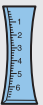

Outcomes: positive result on 2 different HIV tests and adverse effects.

Patient follow up: $91 \%$ (intention to treat analysis).

*See glossary.

tInformation provided by author.

\section{MAIN RESULTS}

The study was stopped early after a third interim analysis. Fewer men in the circumcision group than in the control group contracted HIV

For correspondence: $\operatorname{Dr} R$ C Bailey, University of Illinois at Chicago, Chicago, IL, USA. rcbailey@uic.edu

Sources of funding: National Institute of Allergy and Infectious Diseases and Canadian Institutes of Health Research. infection over the study period (table). Adverse effects occurred in 23 circumcised men; the most common were bleeding and infection ( 5 occurrences each).

\section{CONCLUSION}

In young sexually active men in Kenya, circumcision reduced the risk of contracting HIV infection.

\section{Commentery}

T he study by Bailey et al indicates that adult male circumcision, together with other HIV prevention interventions, reduces HIV acquisition by half. This finding confirms those of many previous observational studies ${ }^{1}$ and 2 other randomised controlled trials. ${ }^{2-3}$ The effect is based on a biologically plausible mechanism of action, which has been suggested by in vitro studies. The strong treatment effect was seen in both observational and intervention studies. The authors document this beneficial effect of circumcision despite sustained increases in self reported safer sexual behaviour, including consistent condom use.

While the Kenyan trial findings provide new options for men, an ongoing trial will examine the potential for circumcision to influence the probability of HIV transmission to women from infected men.

The low rate of adverse events related to circumcision, as performed in a well equipped centre, suggests what may be achieved. A critical aspect of developing public health programmes that include circumcision will be the ability to provide suitable surgical care. Bailey et al speculate whether the higher rates of adverse events observed in another randomised trial in South Africa, in which general practitioners used the same surgical technique in their offices, ${ }^{3}$ may have been a function of the surgical setting and aftercare.

The finding that circumcision was only partially efficacious also indicates that this cannot be seen as a stand-alone intervention but will need thoughtful and sustainable integration into existing prevention approaches.

Guy de Bruyn, MBBCh MPH University of the Witwatersrand Johannesburg, South Africa

1 Siegfried N, Muller M, Volmink J, et al. Male circumcision for prevention of heterosexual acquisition of HIV in men. Cochrane Database Syst Rev 2003;(3):CD003362.

2 Gray RH, Kigozi G, Serwadda D, et al. Male circumcision for HIV prevention in men in Rakai, Uganda: a randomised trial. Lancet 2007; 369:657-66

3 Auvert B, Taljaard D, Lagarde E, et al. Randomized, controlled intervention trial of male circumcision for reduction of HIV infection risk: the ANRS 1265 Trial. PLoS Med 2005;2:e298.

Circumcision $v$ delayed circumcision (control) in young sexually active Kenyan men*

\begin{tabular}{lllll}
\hline Outcomes at median $\mathbf{2 4}$ months & Circumcision & Control & RRR (95\% CI) & NNT (CI) \\
\hline HIV infection & $2.1 \%$ & $4.1 \%$ & $53 \%(22$ to 72$)$ & 45 (34 to 109) \\
\hline
\end{tabular}

*Abbreviations defined in glossary; RRR, NNT, and $\mathrm{Cl}$ calculated from data in article. 